\section{Antibiotische Therapie: Behandlungsdauer häufiger Infekte in der Praxis}

Pouwels KB et al. Duration of antibiotic treatment for common infections in English primary care: cross sectional analysis and comparison with guidelines. BMJ 2019; 364: 1440

Die Resistenz-Entwicklung gegenüber einer antibiotischen Therapie kann u.a. durch eine Reduktion nicht angemessenen Verschreibungsverhaltens reduziert werden. Voraussetzungen hierfür sind eine klare Indikationsstellung, die Berücksichtigung spezifischer Umstände und der Verzicht auf eine unnötig lange Therapiedauer. Fragestellung der vorliegenden Studie war die Verschreibungsdauer bei häufig vorkommenden Infekten verglichen mit den Leitlinien.

Die Querschnitts-Studie griff auf Daten englischer Allgemeinarztpraxen der Grundversorgung von 2013-2015 zurück. Zur Auswertung kamen 931015 Arztkontakte, welche zu einer Verschreibung einer antibiotischen Therapie führten. Indikationen waren

- akute Sinusitis,

- akute Rachenentzündung,

- akuter Husten und Bronchitis,

- Lungenentzündung.

- akute Exazerbation einer chronisch obstruktiven Lungenerkrankung,

- akute Otitis media,

- akute Zystitis,

- akute Prostatitis,

- Pyelonephritis,

- akute Hautinfektionen,

- Impetigo,

- Scharlach und

- Gastroenteritis.

Primäre Fragestellungen waren

- die prozentuale Häufigkeit von antibiotischen Verschreibungen mit einer Behandlungsdauer über die von den Leitlinien empfohlene hinaus und

- die absolute Zahl an Tagen außerhalb der empfohlenen Dauer für die einzelnen Indikationen.
Die häufigsten Gründe für die Verschreibung einer antibiotischen Therapie waren

- akuter Husten und Bronchitis (386972 Personen, 41,6\% der Konsultationen),

- akute Rachenentzündung (239231, $25,7 \%$ ),

- akute Otitis media (83054, 8,9\%) und

- akute Sinusitis (76683, 8,2\%).

Ein Zusammenhang von Verschreibungspraxis und Ko-Morbiditäten oder Lebensalter ließ sich nicht nachweisen. Infekte der oberen Atemwege, akuter Husten und Bronchitis waren verantwortlich für über 2/3 der Verschreibungen. Über 80 \% dieser Behandlungen überschritten in der Behandlungsdauer die LeitlinienEmpfehlungen. Ausnahmen waren akute Sinusitis und akute Rachenentzündung, wo lediglich 9,6\% (95\% Konfidenzintervall $(\mathrm{KI})$ 9,4-9,9) resp. 2,1\% (95\% KI 22,1\%) die empfohlene Behandlungsdauer überschritten.

Bei der akuten Zystitis der Frau lagen über die Hälfte der Verordnungen über der empfohlenen Dauer (54,6\%, 95\% KI $54,1-55)$. Für weitere nicht-respiratorische Infekte war der Prozentsatz mit Überschreitung der empfohlenen Behandlungsdauer niedriger. Insgesamt waren bei 931015 Arztkontakten mit Verordnung einer antibiotischen Therapie an die 1,3 Millionen Tage jenseits der Zeit, welche einer leitliniengerechten Therapiedauer entsprechen würde.

Die Tendenz in der vorliegenden Studie ging zu einer Verschreibungsdauer über 5 oder 7 Tage oder ein Vielfaches davon, 7 Tage waren die häufigste Zeitspanne. Bei Erkrankungsmodalitäten, bei denen die Leitlinien eine längere Therapiedauer empfehlen, war auch die Prozentzahl an darüberhinausgehenden Verordnungen erheblich geringer als bei Indikationen mit nur kurzzeitig empfohlener Therapie. Eine kürzere als empfohlene Therapiedauer zeigte sich bei der akuten Prostatitis mit 52,3\% unter den empfohlenen 28 Tagen und 26\% unter 14 Tagen sowie bei der akuten Zystitis des Mannes mit 31,8\% unter 7 Tagen. Bei den meisten Indikationen entsprachen $\geq 75 \%$ der verordneten antibiotischen Therapeutika den hierfür von den Leitlinien empfohlenen Substanzen. Eine relevante Ausnahme bot die Gastroenteritis, bei der lediglich $12,8 \%$ mit einem der empfohlenen Wirkstoffe behandelt worden waren. Auch anders als bei anderen Indikationen war hier die Therapiedauer betrachtet lediglich für die in den Leitlinien empfohlenen Antibiotika kürzer (40\% vs. 53,3\%).

Die übermäßige Nutzung antibiotischer Therapien fördert, so Pouwels et al., nicht nur die Resistenzentwicklung, sondern auch das Risiko unerwünschter Nebenwirkungen. Diese beinhalten z. B. Diarrhoe, Exantheme oder die Entwicklung einer Candidiasis oder Clostridiumdifficile-Infektion. Ein besseres Verständnis der Gründe für die mangelnde Adhärenz an die Leitlinien könnte dazu führen, Interventionsstrategien zu entwickeln.

FAZIT

Eine erhebliche Zahl antibiotischer Verordnungen bei häufig auftretenden Infekten innerhalb der Grundversorgung in englischen Allgemeinarztpraxen überschreitet die in den Leitlinien empfohlene Behandlungsdauer. Die Anpassung der Dauer einer antibiotischen Therapie an die Leitlinien kann daher - so Pouwels et al. - bereits einen wesentlichen Beitrag leisten, die absolute Verordnungsmenge zu reduzieren und damit Resistenzentwicklungen entgegenzutreten.

Dr. Birgit Gappa, Penzberg

\section{Kommentar}

Ursächlich für die Entwicklung von Antibiotikaresistenzen ist bekanntermaßen vermeidbarer Antibiotikaeinsatz in Massentierhaltung und Humanmedizin. Bisher fokussierten nationale Strategien (in Deutschland DART2020) für die Humanmedizin auf Indikationsstellung und Substanzauswahl mit Schwerpunkt auf der stationären Versorgung - das ändert sich derzeit [1]. Die vorliegende Querschnittsstudie unterstreicht hierzu die Bedeutung der Anwendungsdauer und des ambulanten Sektors. Annehmbar sind die Ergebnisse auch auf das deut- 
sche Gesundheitswesen übertragbar und werden daher das Design zukünftiger Surveillance-Studien [2] und schließlich auch geplante Interventionen beeinflussen [3].

Der Befund, dass $80 \%$ der Verordnungsmengen die Leitlinienempfehlungen übersteigen, ist beeindruckend, auch wenn dieser (nur) auf Sekundärdatenanalysen und nicht auf beobachteter Anwendungsdauer beruht. Hochgerechnet auf ganz Großbritannien würden sich allein aus einer leitliniengerechten Anwendungsdauer der Antibiotikatherapie bei Atemwegs- und Harnwegsinfekten jährlich 10000000 vermeidbare Tagesdosen in menschlichen Körpern und Umwelt ergeben. Im Durchschnitt wären pro Antibiotikatherapie 1,5 Anwendungstage vermeid- und verzichtbar.

Zu den Ursachen und Steuerungsmöglichkeiten dieses Missstandes gehört die notwendige Aktualisierung unserer Wissensvorräte: So sind heute „Fünf Tage Cotrim“ für unkomplizierte Harnwegsinfekte oder „Zehn Tage Oral-Penicillin“ bei Tonsillitis obsolet, wurden aber von der Mehrzahl der heute niedergelassenen Ärzte noch so gelernt. Deshalb geht es nicht nur um geeignete und verständliche Leitlinien zu Antibiotikatherapien, sondern auch um deren zielgruppengerechte Verbreitung und Implementierung. Was hierzu im Krankenhaus die „Antibiotic Stewardships“ sind, werden im ambulanten Bereich Qualitätszirkel und vielleicht einmal Peer-Vergleiche als Anteile verhaltensändernder Interventionen werden [4]. Dabei wird idealerweise das gesamte Praxisteam Adressat, denn die Hausarztpraxis der Zukunft wird mehr Aufgaben zur Patientenkommunikation und Qualitätssicherung auf nicht-ärztliche Gesundheitsberufe übertragen [5].

Die zeitliche Verkürzung von Antibiotikatherapien ist weniger aufwändig über Information und Gespräch mit den Patien- ten umzusetzen als deren Vermeidung; deshalb ist die vorliegende Studie so bedeutsam. Trotzdem: Weniger Rezepte schreiben, kostet im Zweifel mehr Gesprächszeit. Dafür müssen wir die Bedingungen in unserem Gesundheitssystem und unseren Praxen entsprechend gestalten.

\section{Autorinnen/Autoren

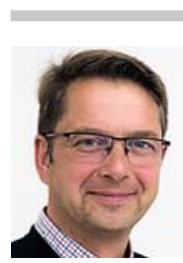 \\ Prof. Dr. med \\ Andreas Klement \\ ist Facharzt für Allgemein- medizin und Chirurgie. Als niedergelassener Haus- arzt arbeitet er in Teilzeit als Ärztlicher Manager im Kompetenzzentrum für die Weiterbildung Allgemein- medizin der Universität Halle-Wittenberg}

\section{Literatur}

[1] Bundesministerium für Gesundheit. DART 2020: Dritter Zwischenbericht 2018 (April 2018). Im Internet: https://www.bundesgesundheitsministerium.de/fileadmin/Dateien/ 3_Downloads/D/DART_2020/ BMG_DART2020_3-Zwischenbericht_2018_DT.PDF; Stand 23.03.19

[2] Zweigner J, Meyer E, Gastmeier P et al. Rate of antibiotic prescriptions in German outpatient care - are the guidelines followed or are they still exceeded? GMS Hyg Infect Control 2018; 13: Doc04. doi:10.3205/dgkh000310

[3] Bätzing-Feigenbaum ], Schulz M, Schulz M et al. Outpatient Antibiotic Prescription. Dtsch Arztebl Int 2016; 113: 454-459. doi:10.3238/arztebl.2016.0454

[4] Meeker D, Linder JA, Fox CR et al. Effect of Behavioral Interventions on Inappropriate Antibiotic Prescribing Among Primary Care Practices: A Randomized Clinical Trial. JAMA 2016; 315: 562 - 570. doi:10.1001/jama.2016.0275

[5] Wagner EH, Flinter M, Hsu C et al. Effective team-based primary care: observations from innovative practices. BMC Fam Pract 2017; 18 : 13. doi:10.1186/s12875-017-0590-8 